\title{
Synchronous and metachronous metastasis to renal parenchyma of esophageal squamous cell carcinoma: two case reports and review of the literature
}

\author{
Jun Jia $^{1 \wedge}$, Wei Sun ${ }^{2}$, Dongmei Lin ${ }^{2}$, Xiaodong Zhang ${ }^{1 \wedge}$ \\ ${ }^{1}$ Key Laboratory of Carcinogenesis and Translational Research (Ministry of Education/Beijing), the VIP-II Division of Medical Department, Peking \\ University Cancer Hospital \& Institute, Beijing, China; 'Key Laboratory of Carcinogenesis and Translational Research (Ministry of Education/ \\ Beijing), Department of Pathology, Peking University Cancer Hospital \& Institute, Beijing, China \\ Correspondence to: Dr. Xiaodong Zhang. Chief Physician, VIP-II Division of Medical Department, Peking University Cancer Hospital \& Institute, \\ No. 52 Fucheng Road, Haidian District, Beijing 100142, China. Email: bdzlvip@163.com.
}

\begin{abstract}
Metastasis of esophageal squamous cell carcinoma (ESCC) spread to uncommon sites is increasing in recent years. Metachronous renal metastasis of ESCC was reported before and relatively easy to be diagnosed. But synchronous renal parenchyma metastasis of ESCC is rare and of importance before make treatment plan. Here we present one case of synchronous and one case of metachronous renal parenchyma of ESCC. In the synchronous renal metastasis case, the patient was diagnosed ESCC in August 2016. Though CT scan was normal, PET-CT scan found a small lesion in right renal cortex with increased FDG uptake. The patient refused biopsy of renal lesion. Two cycles of neoadjuvant chemotherapy were given with stable response. Then esophagectomy and adjuvant radiation were given. Seven months later, CT scan revealed renal and spleen lesions and biopsy confirmed squamous cell carcinoma. The patient refused systematic treatment and died 5 months later. In the metachronous renal metastasis case, the patient was diagnosed ESCC in March 2015 and PET/CT scan showed no distant metastasis. Esophagectomy and adjuvant radiotherapy were given. CT scan revealed solitary renal and multiple lung metastasis 23 months later. Biopsy of renal lesion confirmed squamous cell carcinoma (SCC). Chemotherapy was given but with only stable response. The patient died 7 months later after relapse. Both patients had not urinary symptoms. Together with published literatures, renal parenchyma metastasis of ESCC is often asymptomatic. Disease history and biopsy are helpful in diagnosis. And present cases suggested that PET-CT is more sensitive than CT to identify unusual metastasis of ESCC.
\end{abstract}

Keywords: Esophageal squamous cell carcinoma (ESCC); metastasis; kidney; case report

Submitted May 24, 2020. Accepted for publication Dec 12, 2020.

doi: $10.21037 /$ tcr-20-2167

View this article at: http://dx.doi.org/10.21037/tcr-20-2167

\section{Introduction}

Esophageal cancer is an aggressive disease and ranks the 6th cause of cancer death worldwide. Different from the relatively high incidence of adenocarcinoma, the squamous cell carcinoma (SCC) is the major pathological type of esophageal cancer in Asian countries. The 5-year survival rate of esophageal squamous cell carcinoma (ESCC) is about $40 \%$ for local-regional disease and less than $5 \%$ for metastatic disease (1). The incidence of distant metastases at newly diagnosed ESCC is reported about $20 \%$ to $30 \%$. But this

^ ORCID: Jun Jia, 0000-0002-1644-8075; Xiaodong Zhang, 0000-0001-9906-8555. 
proportion increases to about $40-50 \%$ in recurrent disease after radical esophagectomy (2) or definitive concurrent radio-chemotherapy (3) in ESCC. The most common sites of distant metastasis are liver, lung, bone, adrenal glands and sometime brain in ESCC (4). However, unexpected metastasis spread to uncommon sites in ESCC has been increasingly reported in recent years. In a systematic review, 164 patients with unexpected metastasis of esophageal cancer were identified by searching 10,024 articles published between 1982 and February, 2017. And 10 (6.0\%) of the 164 cases of unexpected metastasis were found to be renal metastasis (5). When review literature, renal metastasis of ESCC were almost metachronous and many diagnosed after nephrectomy $(6,7)$. Here, we present a case of synchronous renal parenchyma metastasis of ESCC and a case of metachronous one including their clinical, imaging, and pathological features. We hope it would be helpful for clinicians to identify unusual metastasis of ESCC in certain individual cases before making treatment plan. We present the following case in accordance with the CARE reporting checklist (available at http://dx.doi. org/10.21037/tcr-20-2167).

\section{Case presentation}

Case 1 (synchronous renal metastasis): the patient was a 66-year-old man. He felt progressive choke for 3 months and consulted doctor in August 2016. The patient had no family and other medical history. Physical examination on admission was almost normal. Laboratory tests showed increased serum neuron-specific enolase (NSE) of 29.0 (normal range, 0-15.2) ng/mL. Endoscopy revealed multiple polypoidal-looking tumors in the whole thoracic esophagus (20-22, 23-31, 32-37 cm from the incisor teeth). The pathological diagnosis was poor differentiated squamous cell carcinoma in biopsy samples of all three esophageal lesions. A FDG-PET/CT scan with attenuation showed significantly increased uptake of FDG in the primary esophageal lesion (SUVmax, 21.4) (Figure 1A,B,C,D), mediastinal lymph nodes (SUVmax, 2.9), and also a small lesion in right renal cortex (SUVmax, 9.4) (Figure 1E,F). Further CT scan confirmed the thickened esophageal wall of $28 \mathrm{~mm}$ and multiple enlarged mediastinal and paracardial lymph nodes with the largest one of $18 \times 10 \mathrm{~mm}$ but normal kidney (Figure 1G). The patient was diagnosed ESCC, advanced stage of $\mathrm{cT}_{3} \mathrm{~N}_{1} \mathrm{Mx}$. Biopsy of renal lesion was suggested but the patient refused. This patient was given two cycles' chemotherapy of cisplatin and paclitaxel. There was only stable response. After communication with the patient, according to his wishes, this patient received esophagectomy of primary tumor and lymphadenectomy in October 2016. The pathological stage was $\mathrm{pT}_{3} \mathrm{~N}_{2}$ (4 of 33 metastatic lymph nodes). Adjuvant radiotherapy of mediastinum with a dosage of GTV $50 \mathrm{~Gy} / 25 \mathrm{f}$ was given after surgery in December 2016. In surveillance (7 months after PET/CT), a CT scan revealed a solitary tumor of $15 \times 16 \mathrm{~mm}$ in right renal parenchyma (Figure $1 \mathrm{H}$ ) and also a tumor of $53 \times 40 \mathrm{~mm}$ in the spleen (Figure 1I). And NSE level increased to be of $106.7 \mathrm{ng} / \mathrm{mL}$. Biopsy of the renal tumor confirmed squamous cell carcinoma (Figure 2). Chemotherapy was recommended. But the patient refused. One month later, the patient complained headache. Further CT scan confirmed brain metastasis. Radiation of brain was given and there were no severe adverse events. Then the patient received palliative treatment and died 5 months later in September 2017.

Case 2 (metachronous renal metastasis of ESCC): the patient was a 60 -year-old man. He suffered from choke and weight-loss for 4 months and counselled to doctor in March 2015. The patient also had no family and other medical history. Endoscopy revealed an ulcer-looking tumor with an irregular border in the lower thoracic esophagus, $35-40 \mathrm{~cm}$ from the incisor teeth. The pathological diagnosis was poor differentiated squamous cell carcinoma in biopsy sample of esophageal lesion. Laboratory tests also showed increased serum NSE of 16.82 (normal range, 0-15.2) ng/mL. A FDG-PET/CT scan with attenuation showed significantly increased uptake of FDG in the esophageal lesion with the SUVmax of 9.56, paraoesophageal lymph node with SUVmax of 3.2, slightly increased uptake of FDG in the right supraclavicular and para-stomach lymph nodes with the SUVmax of 0.7-1.7 (Figure 3A,B,C,D), and normal kidney (Figure 3E,F). A CT scan confirmed the thickened esophageal wall of $13 \mathrm{~mm}$, multiple enlarged lymph nodes with the largest one of $10 \times 6 \mathrm{~mm}$ in the lesser curvature of stomach, and also normal kidney (Figure 3G). This patient received esophagectomy in April 2015 and the pathological stage was pT3N1 (1/22 metastatic lymph nodes). After surgery, the patient was given adjuvant radiotherapy of the mediastinum and supraclavicular area with the GTV dosage of $56 \mathrm{~Gy} / 28 \mathrm{f}$ in May 2015. After a regular surveillance of 23 months, in March 2017, a CT scan showed multiple tumors in lung (Figure $3 \mathrm{H}$ ) and a solitary tumor of $48 \mathrm{~mm}$ in diameter in the left renal cortex (Figure 3I,7). Biopsy of the renal tumor confirmed poor differentiate squamous cell carcinoma with the immunochemical staining of CK5/6(+), P40(+), P63(+), CA9(-), PAX2(-), PAX8(-) (Figure 4). Then 


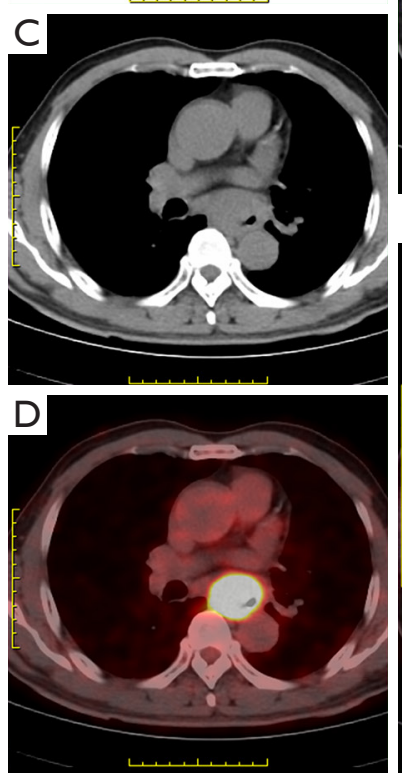

A

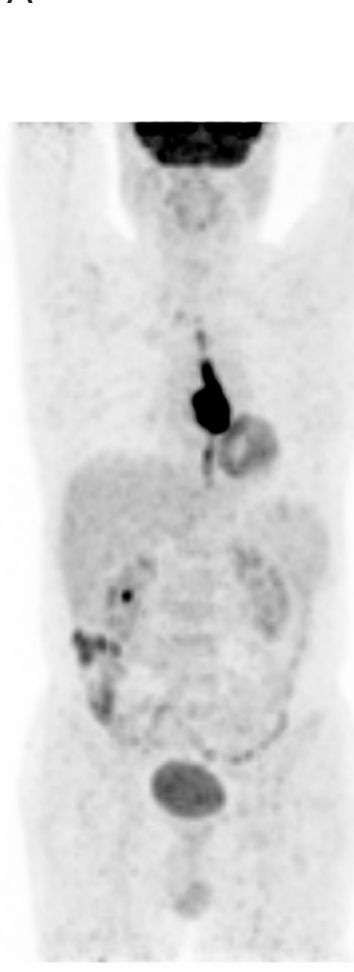

B

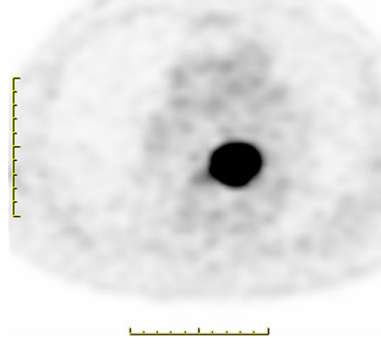

\section{E}


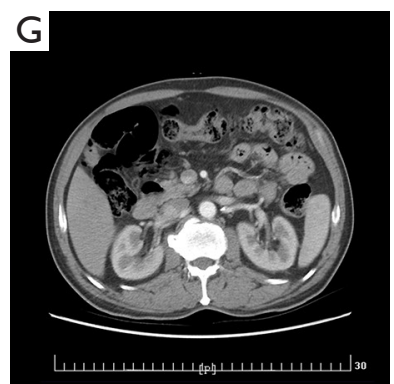

$\mathrm{H}$
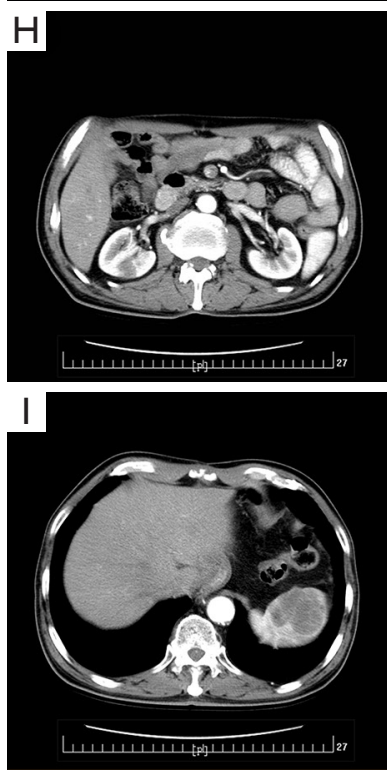

Figure 1 CT and PET-CT scan images of primary tumor before esophagectomy and metastatic tumors when relapse in case 1: synchronous renal metastasis of ESCC. PET-CT scan images of body (A), primary tumor of esophagus (B,C,D), and a small right renal parenchyma lesion (E,F) with increased uptake of ${ }^{18} \mathrm{~F}$-FDG. CT scan image of normal kidney (G) at diagnosis, and metastatic tumors of right renal parenchyma (H) and spleen (I) 5 months after esophagectomy. The scale was $1 \mathrm{~cm}$. ESCC, esophageal squamous cell carcinoma.

the patient was given chemotherapy of cisplatin, paclitaxel and anti-EGFR agent nimotuzumab. Disease remained stable after two cycles' treatment. The patient refused to further chemotherapy owing to gastrointestinal adverse events and died in October 2017.

The development of disease and treatment of present two cases had been summarized in Figure 5.

All procedures performed in studies involving human participants were in accordance with the ethical standards of the institutional and/or national research committee(s) and with the Helsinki Declaration (as revised in 2013). Written informed consent was obtained from the patient.

\section{Discussion}

Though it was reported that $1.8-12.6 \%$ of patients dying of cancers had renal metastases (8), the diagnosis of renal metastasis of esophageal cancer is relatively difficult in clinic. Firstly, it is reported that squamous cell carcinoma of the upper urinary tract is account for $10 \%$ of all renal pelvic tumors and $0.5 \%$ of all renal malignancies (9). Secondly, there is no clinical, imaging, morphological, and histopathological features to distinguish a metastatic SCC from a primary one of kidney. Hematuria is one of the most common symptoms in primary renal cancer, but it occur mainly in advanced disease of renal cancer (10). Hematuria was reported a symptom of ESCC renal metastasis mostly in literatures before 2000 when contract CT as a method for staging and surveillance was not routinely performed $(7,11)$. Thereafter, the renal metastasis of ESCC is often asymptomatic and diagnosed by CT scan $(6,12,13)$. In the present two cases, both of them were without hematuria 

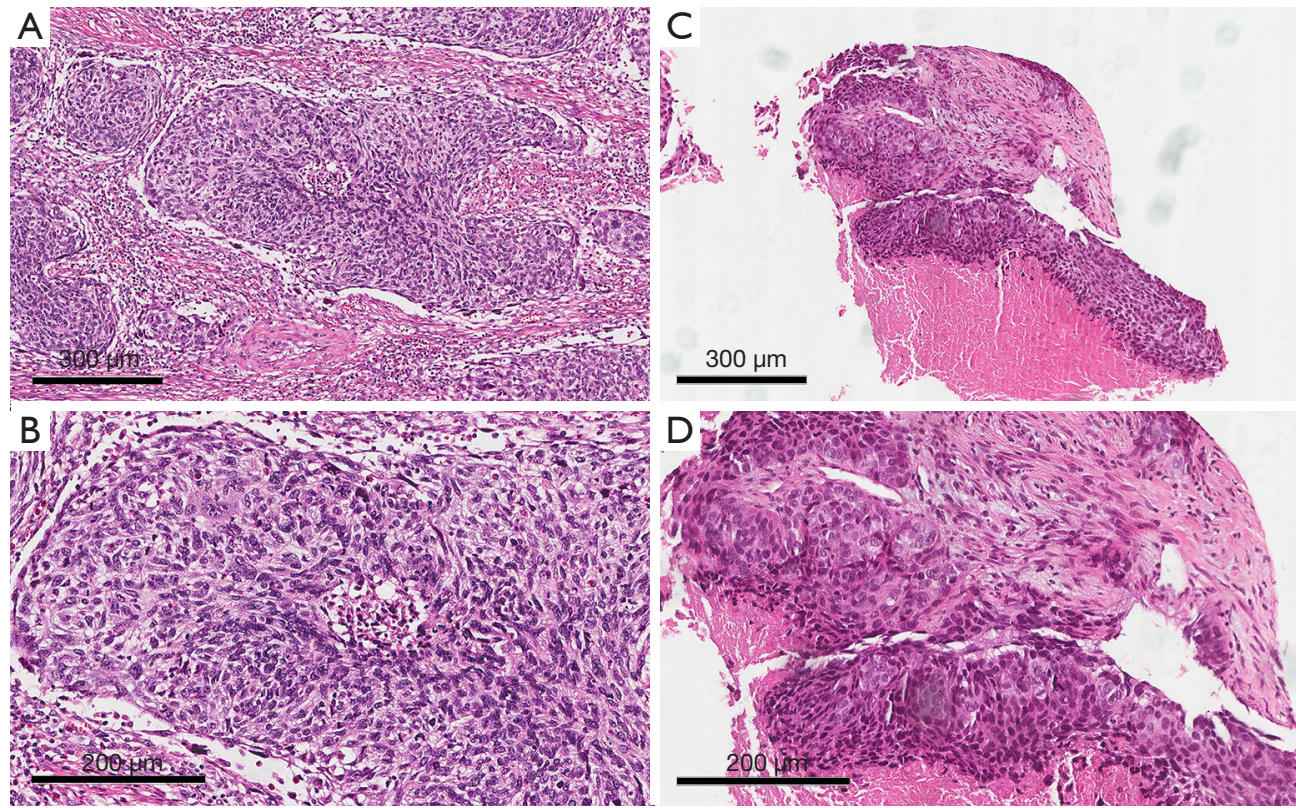

Figure 2 Pathological image of primary tumor and renal metastatic tumors in case 1: synchronous renal metastasis of ESCC. Hematoxylineosin staining of primary tumor (A,C) and renal metastatic tumor (B,D) under light microscope of 100 times (A,B) and 200 times (C,D). Scale bars were labelled on each picture respectively. ESCC, esophageal squamous cell carcinoma.

and proteinuria even till the death of patients.

CT scan could find tumor in renal and is the recommended staging methods of esophageal cancer now. But there are no distinguished CT appearances of metastatic tumor lesions in renal parenchyma compared with a primary tumor. The primary renal cancer lesion often appears as solitary infiltrative tumor, especially in early stage (10). As showed in present two cases, metastatic lesion often appears as a solitary tumor infiltrating the renal parenchyma in CT images. So, it is difficult to differ a metastatic tumor from a primary renal cancer simply via CT image.

In literatures, some patients were given nephrectomy and thereafter diagnosed renal metastasis of $\operatorname{ESCC}(6,7,12-14)$. Table 1 summarized some published literatures. But in recent years, biopsy of renal lesion could make diagnosis before give more aggressive interventions. In present synchronous renal metastasis case (case 1), PET-CT reported a small right renal lesion when staging. It was possible that it was caused by other renal disease. Biopsy of renal lesion was suggested but the patient refused. Unfortunately, the renal lesion was finally confirmed to be metastatic SCC by biopsy when CT scan revealed spleen and renal lesions 7 months later. Because cancer cells of SCC derived from different organs have similar morphological appearance. In this situation, immunohistochemistry is another useful method for diagnosis. Immunohistochemical markers, such as CK5/6, P63, and P40 etc., are relative specific biomarkers to identify squamous cell carcinoma. In the present case of metachronous renal metastasis of ESCC (case 2), the metastatic renal tumor was CK5/6(+), P63(+), and P40(+). And staining of CD10, PAX2, and PAX8, which were common biomarkers of renal carcinoma (15), excluded the diagnosis of primary urothelial cancer. But, unfortunately, there are still no specific markers to identify SCC derived from different organs.

In present two cases, the history of ESCC disease and evidence of other metastatic sites were crucial factors to diagnose renal metastasis. In the case 1 , synchronous renal metastasis of ESCC, CT scan revealed concurrent spleen metastatic lesions 7 months after PET/CT. In case 2, metachronous renal metastasis of ESCC, CT scan also revealed concurrent lung metastasis.

Another helpful method for diagnosis of renal metastasis was PET/CT. Previously investigations had demonstrated that $\mathrm{PET} / \mathrm{CT}$ is more sensitive to find distant metastasis of ESCC (16). And whole-body PET or PET/CT is recommended for staging of esophageal cancer in many guidelines. But, the cost of PET and PET/CT is much 
A

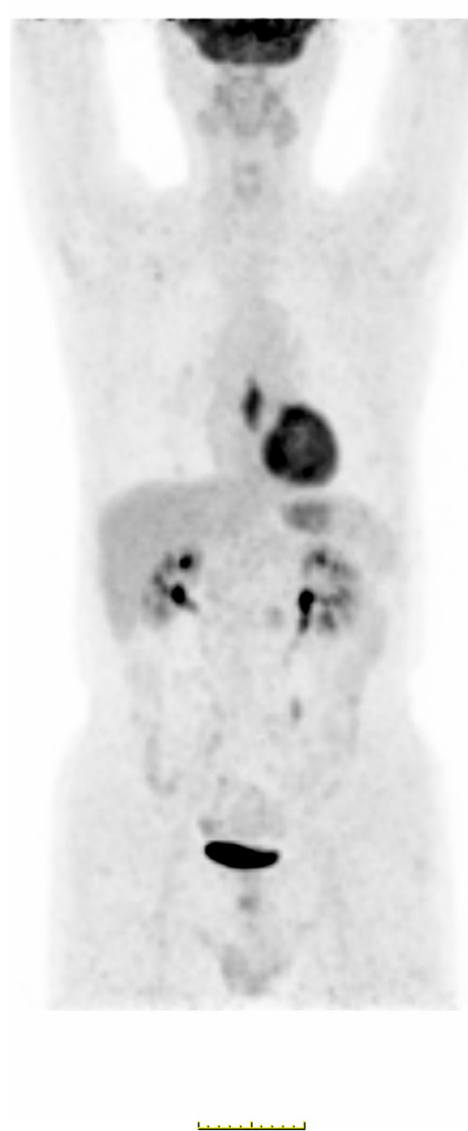

B
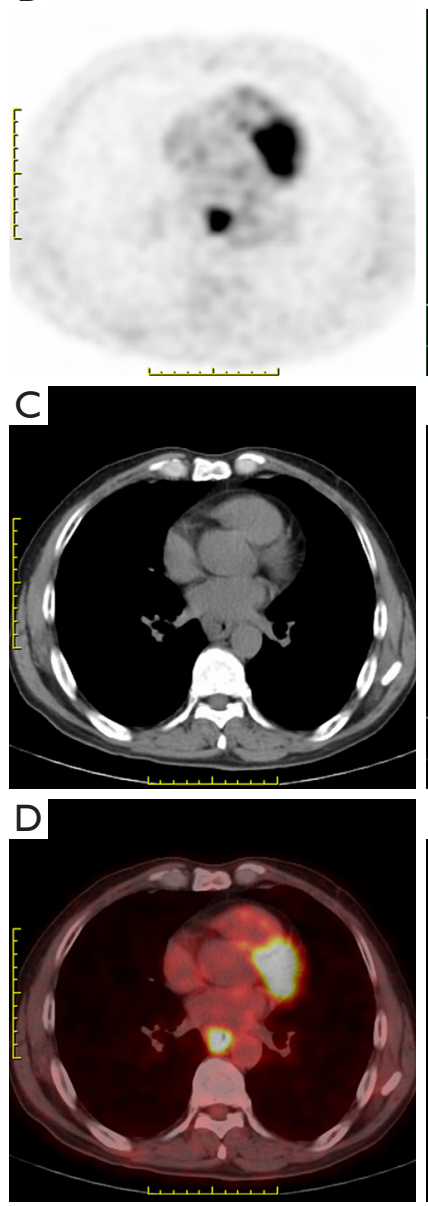
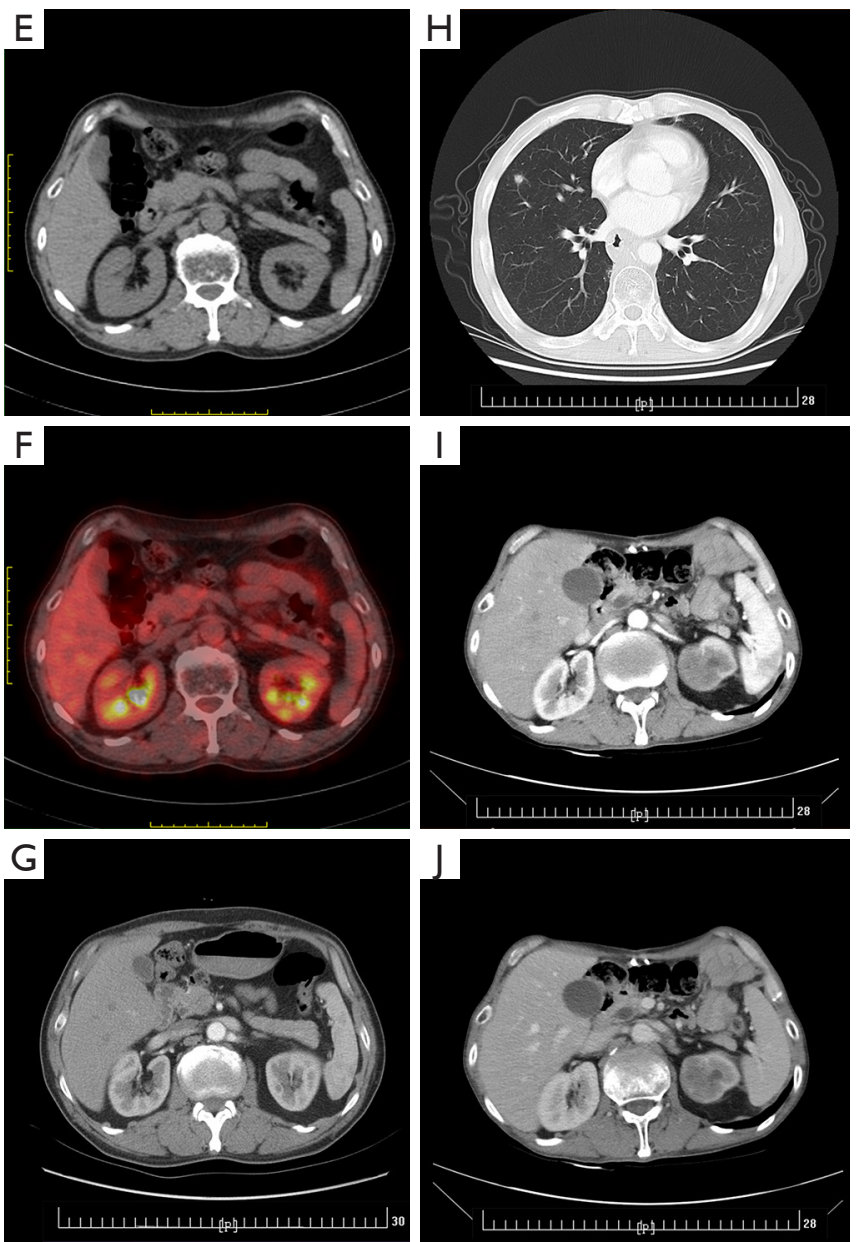

Figure 3 CT and PET-CT scan images of primary tumor and normal kidney before esophagectomy and metastatic tumors when disease progression in case 2: metachronous renal metastasis of ESCC. PET-CT scan images of body (A), primary tumor of esophagus (B,C,D), and normal kidney (E,F) at diagnosis of ESCC. CT scan images of normal kidney $(\mathrm{G})$ at diagnosis and metastatic tumors of lung $(\mathrm{H})$, and left renal parenchyma (I,J) when disease relapse 23 months after esophagectomy. The scale was $1 \mathrm{~cm}$. ESCC, esophageal squamous cell carcinoma.

higher than CT which limited their use in many hospitals, especially in countryside areas of Asian countries. In literatures, previous reports of ESCC renal metastasis were almost metachronous and found by CT scan. In the present case 1 (synchronous renal metastasis of ESCC), though the initial CT scan showed both kidneys was normal, a small solitary lesion with increased FDG intake (SUVmax, 2.3) in renal parenchyma was found in PET/CT scan images. The lesion was identified by CT scan almost 7 months later. If using more sensitive PET or PET/CT to staging, it would be helpful to find synchronous metastasis of unusual sites including kidney. Therefore, we suggested $\mathrm{PET}$ or PET/CT scan should be under consideration when there is suspected metastatic lesions or before giving a radical treatment such as esophagectomy or definitive chemoradiotherapy.

There was one interesting thing in two present cases. Both patients had increased NSE serum level. Especially in presented case 1, synchronous renal metastasis of ESCC, the level of serum NSE was $29.0 \mathrm{ng} / \mathrm{mL}$ at initial diagnosis and it reached to $107.6 \mathrm{ng} / \mathrm{mL}, 7$ times of uppernormal limit, when CT scan revealed renal and spleen metastasis 7 months later. It was reported that NSE is a relatively specific tumor marker of small cell carcinoma or neuroendocrine carcinoma (17). Small cell carcinoma of esophagus is highly progressive. Higher serum NSE level 


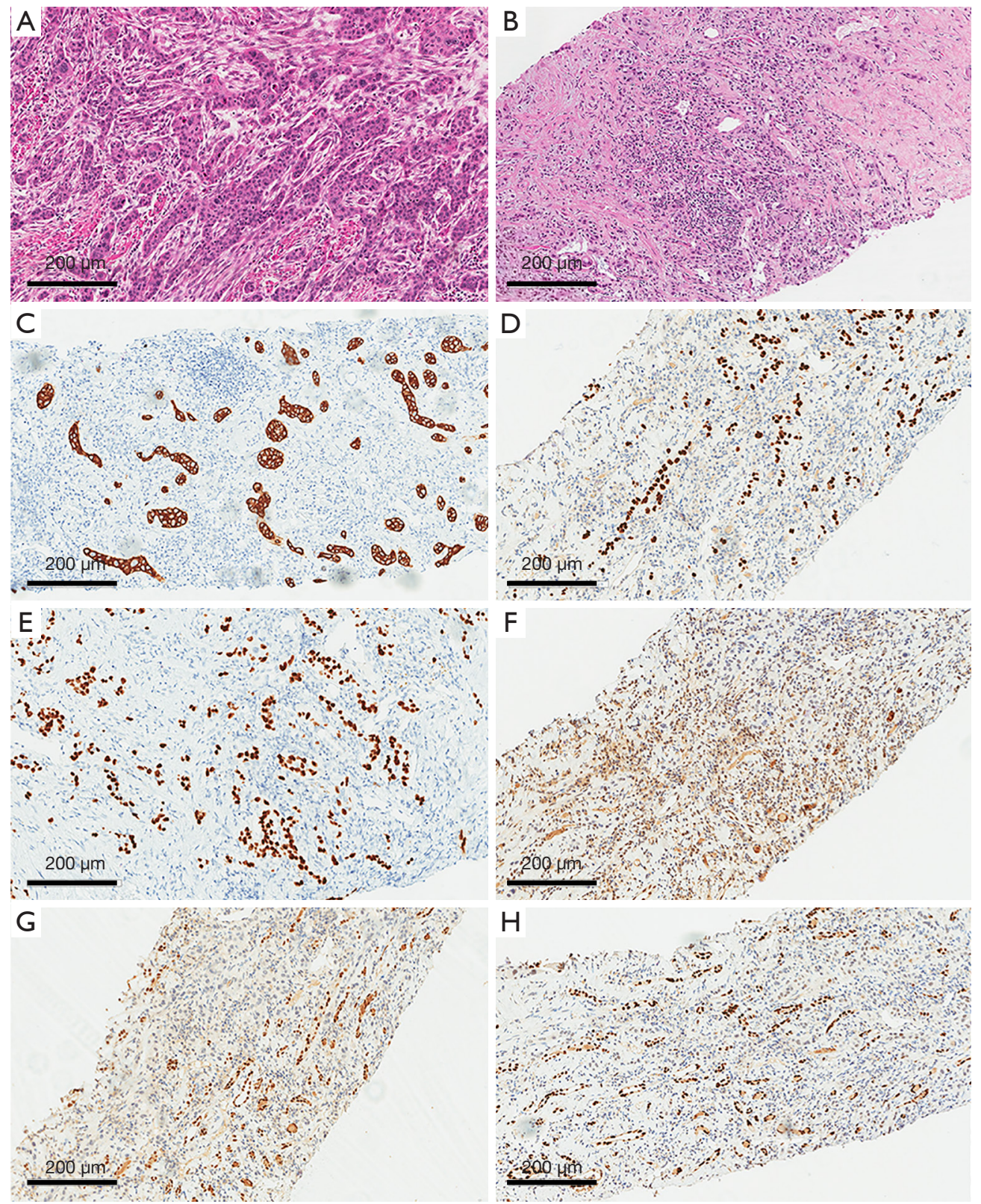

Figure 4 Immunochemical staining of primary and metastatic renal tumors in case 2: metachronous renal metastasis of ESCC. Hematoxylineosin staining of primary tumor (A) and renal metastatic tumor (B) under light microscope of 100 times. Makers of squamous cell carcinoma including cytokeratin 5/6 (C), P40 (D), and P63 (E) were positive while markers of renal cancer including CA9 (F), PAX2 (G), and PAX8 (H) were negative in renal metastatic tumor by immunochemical staining. The scale was $200 \mu \mathrm{m}$. ESCC, esophageal squamous cell carcinoma.

was reported that was related to poor prognosis of small cell carcinoma of esophagus (18). And squamous cell carcinoma mixed of small cell carcinoma of esophagus was also reported (19). But there was no report of increased NSE in ESCC. And in both presented cases, biopsy of primary tumor and metastatic tumor all claimed to be only squamous cell carcinoma. We wondered if there was a possibility that increased serum NSE indicated a more progressive ESCC. Thus, the increased NSE level of esophageal cancer might be of notice in clinic. Detail staging examinations including PET should be under consideration to exclude distant micro-metastasis. 
Case 1

Synchronous renal metastasis of ESCC

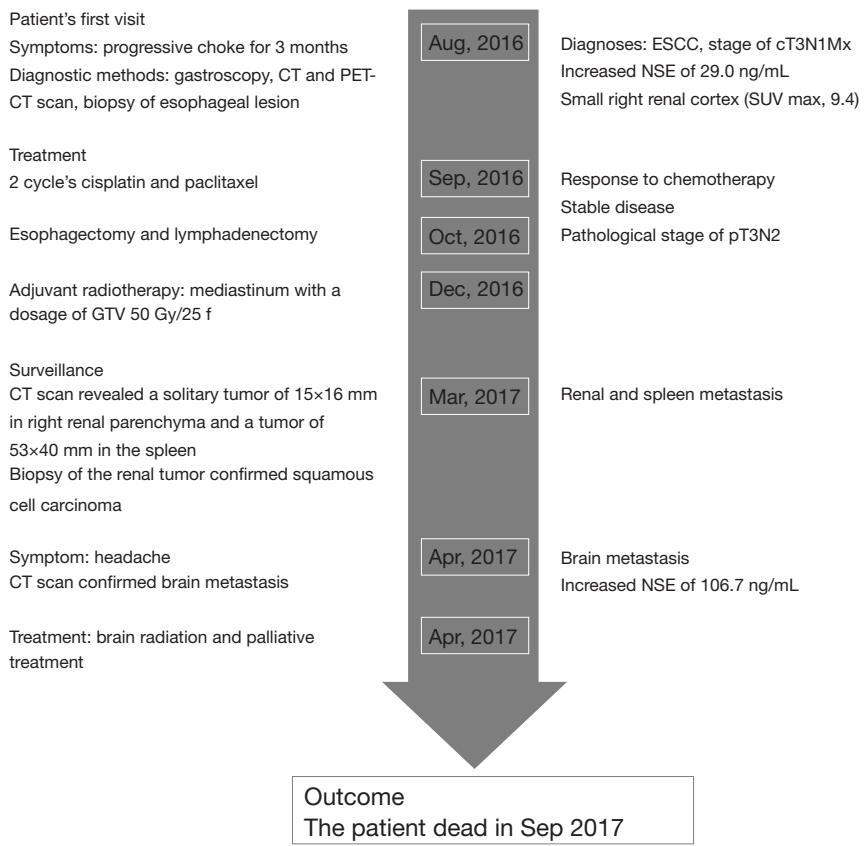

Case 2

Metachronous renal metastasis of ESCC

Patient's first visit

Symptoms: choke and weight-loss for 4 months Mar, 2015 Diagnoses: ESCC, stage of cT3N1MO

Diagnostic methods: gastroscopy, CT and PET-

CT scan, biopsy of esophageal lesion

Treatment

Esophagectomy and lymphadenectomy

Adjuvant radiotherapy: mediastinum with

dosage of GTV 56 Gy/28 $f$

Surveillance

CT scan revealed multiple tumors in lungs and solitary tumor of $48 \mathrm{~mm}$ in diameter in the left renal cortex

Biopsy of the renal tumor confirmed squamous

cell carcinoma

Treatment

Chemotherapy of cisplatin, paclitaxel, and an anti-EGFR antibody nimotuzumab

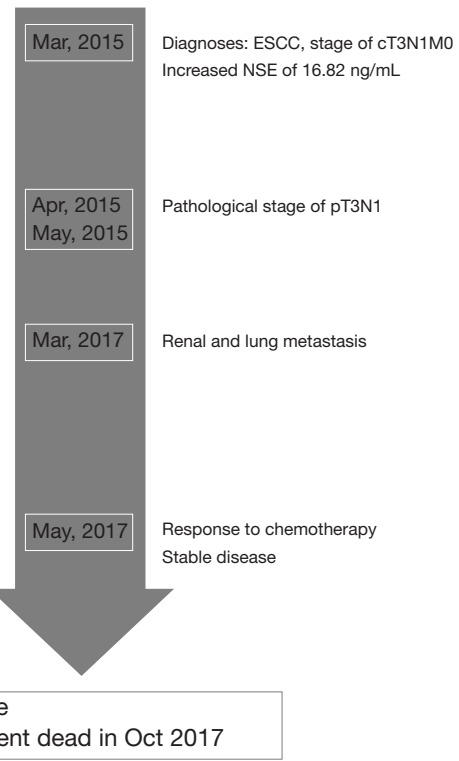

Figure 5 Timeline of present cases. The timeline presents development of disease and treatment of present two cases. ESCC, esophageal squamous cell carcinoma; NSE, neuron-specific enolase.

Table 1 Summary of reported cases of esophageal cancer with renal metastasis

\begin{tabular}{|c|c|c|c|c|c|c|c|c|}
\hline Author & Year & Initial diagnosis & $\begin{array}{l}\text { Stage of primary } \\
\text { tumor }\end{array}$ & $\begin{array}{c}\text { Interval between } \\
\text { esophagectomy and renal } \\
\text { metastasis (months) }\end{array}$ & Symptoms & $\begin{array}{l}\text { Diagnostic } \\
\text { methods }\end{array}$ & $\begin{array}{l}\text { Other } \\
\text { metastatic } \\
\text { sites }\end{array}$ & Treatment \\
\hline $\begin{array}{l}\text { Mikata } \\
\text { et al. (7) }\end{array}$ & 1990 & ESCC & III, TxNOMO & 17 & Hematuria & $\mathrm{CT}$, path & None & Nephrectomy \\
\hline $\begin{array}{l}\text { Matsushita } \\
\text { et al. (12) }\end{array}$ & 1998 & ESCC & Unknown & 13 & None & $\mathrm{CT}$, path & None & Nephrectomy \\
\hline $\begin{array}{l}\text { Lim } \\
\text { et al. (13) }\end{array}$ & 2004 & ESCC & урTONOMO & 23 & None & CT, path & None & $\begin{array}{l}\text { Nephrectomy } \\
\text { and } \\
\text { chemotherapy }\end{array}$ \\
\hline
\end{tabular}

ESCC, esophageal squamous cell carcinoma; CT, computed tomography; path, pathology; EC, esophageal cancer. 
As recommended, treatment for metastasis disease of ESCC is systematic treatment. In present case 2 (metachronous renal metastasis of ESCC), the patient received two cycles' chemotherapy of paclitaxel, cisplatin and anti-EGFR agent nimotuzumab treatment based on promising results of one of our phase 2 clinical trial (20). Disease remain stable after two cycles' treatment and progressed 4 months later. In other reports, cytotoxic agents such as cisplatin, paclitaxel, $5-\mathrm{Fu}$, and gemcitabine were investigated to treat renal metastasis of ESCC, but the clinical response is still poor and most patients died in 6-8 months.

The present cases also had some limitations. Firstly, continuous pathology slice would be helpful to exclude mixed small cell carcinoma in case of increased serum NSE level. Secondly, genomic signature could provide detail molecular characteristics of cancer cells. Comparing genomic signatures of both primary cancer and metastatic tumor might be also useful to exclude renal primary squamous cell carcinoma.

\section{Conclusions}

Renal parenchyma metastasis of ESCC is rare and asymptomatic in clinic. PET or PET/CT is helpful to find some synchronous metastasis of ESCC. It is still difficult to distinguish a renal metastatic tumor from a primary one by either CT scan or biopsy. History of ESCC and evidences of other concurrent metastasis were much helpful for diagnosing. Systematic treatment is recommended for renal metastasis of ESCC, but the prognosis is still poor.

\section{Acknowledgments}

We acknowledge all staff in the VIP-II GI division for their help in collection of data.

Funding: None.

\section{Footnote}

Reporting Checklist: The authors have completed the CARE reporting checklist. Available at http://dx.doi.org/10.21037/ tcr-20-2167

Conflicts of Interest: All authors have completed the ICMJE uniform disclosure form (available at http://dx.doi. org/10.21037/tcr-20-2167). The authors have no conflicts of interest to declare.

Ethical Statement: The authors are accountable for all aspects of the work in ensuring that questions related to the accuracy or integrity of any part of the work are appropriately investigated and resolved. All procedures performed in studies involving human participants were in accordance with the ethical standards of the institutional and/or national research committee(s) and with the Helsinki Declaration (as revised in 2013). Written informed consent was obtained from the patient.

Open Access Statement: This is an Open Access article distributed in accordance with the Creative Commons Attribution-NonCommercial-NoDerivs 4.0 International License (CC BY-NC-ND 4.0), which permits the noncommercial replication and distribution of the article with the strict proviso that no changes or edits are made and the original work is properly cited (including links to both the formal publication through the relevant DOI and the license). See: https://creativecommons.org/licenses/by-nc-nd/4.0/.

\section{References}

1. Siegel RL, Miller KD, Jemal A. Cancer statistics, 2016. CA Cancer J Clin 2016;66:7-30.

2. Yamashita K, Watanabe M, Mine S, et al. Patterns and outcomes of recurrent esophageal cancer after curative esophagectomy. World J Surg 2017;41:2337-44.

3. Sudo K, Kato K, Kuwabara H, et al. Patterns of relapse after definitive chemoradiotherapy in stage II/III (non-T4) esophageal squamous cell carcinoma. Oncology 2018;94:47-54.

4. Quint LE, Hepburn LM, Francis IR, et al. Incidence and distribution of distant metastases from newly diagnosed esophageal carcinoma. Cancer 1995;76:1120-5.

5. Shaheen O, Ghibour A, Alsaid B. Esophageal cancer metastases to unexpected sites: a systematic review. Gastroenterol Res Pract 2017;2017:1657310.

6. Sun Y, Yu X, Zhang Y. Renal metastasis after esophagectomy of esophageal squamous cell carcinoma: a case report and literature review. World J Surg Oncol 2014;12:165.

7. Mikata N, Imao S, Katoh A, et al. Esophageal cancer metastatic to the kidney: report of a case. Nihon Gan Chiryo Gakkai Shi 1990;25:1492-6.

8. Bailey JE, Roubidoux MA, Dunnick NR. Secondary renal 
neoplasms. Abdom Imaging 1998;23:266-74.

9. Busby JE, Brown GA, Tamboli P, et al. Upper urinary tract tumors with nontransitional histology: a single-center experience. Urology 2006;67:518-23.

10. Lewis G, Maxwell AP. Early diagnosis improves survival in kidney cancer. Practitioner 2012;256:13-6, 2.

11. Marsan RE, Baker DA, Morin ME. Esophageal carcinoma presenting as a primary renal tumor. J Urol 1979;121:90-1.

12. Matsushita Y, Katoh T, Isurugi K, et al. Metastatic renal tumor originating from esophageal carcinoma: a case report. Hinyokika Kiyo 1998;44:591-4.

13. Lim DH, Im YH, Ji SH, et al. Esophageal squamous cell carcinoma recurring as a solitary renal mass. Cancer Res Treat 2004;36:271-4.

14. Chang KP, Huang CP, Chang H. Solitary renal metastasis of esophageal squamous cell carcinoma mimicking primary renal neoplasm - a case report and literature review. Biomedicine (Taipei) 2016;6:6.

15. Gailey MP, Bellizzi AM. Immunohistochemistry for the novel markers glypican $3, \mathrm{PAX} 8$, and p40 $(\Delta \mathrm{Np} 63)$ in

Cite this article as: Jia J, Sun W, Lin D, Zhang X. Synchronous and metachronous metastasis to renal parenchyma of esophageal squamous cell carcinoma: two case reports and review of the literature. Transl Cancer Res 2021;10(2):1135-1143. doi: $10.21037 /$ tcr-20-2167 squamous cell and urothelial carcinoma. Am J Clin Pathol 2013;140:872-80.

16. Bruzzi JF, Truong MT, Macapinlac H, et al. Integrated CT-PET imaging of esophageal cancer: unexpected and unusual distribution of distant organ metastases. Curr Probl Diagn Radiol 2007;36:21-9.

17. Isgrò MA, Bottoni P, Scatena R. Neuron-specific enolase as a biomarker: biochemical and clinical aspects. Adv Exp Med Biol 2015;867:125-43.

18. Yan H, Wang R, Jiang S, et al. NSE can predict the sensitivity to definitive chemoradiotherapy of small cell carcinoma of esophagus. Med Oncol 2014;31:796.

19. Yun JP, Zhang MF, Hou JH, et al. Primary small cell carcinoma of the esophagus: clinicopathological and immunohistochemical features of 21 cases. BMC Cancer 2007;7:38.

20. Lu M, Wang X, Shen L, et al. Nimotuzumab plus paclitaxel and cisplatin as the first line treatment for advanced esophageal squamous cell cancer: a single centre prospective phase II trial. Cancer Sci 2016;107:486-90. 